\title{
ON THE GEOMETRY OF THE UNIT SPHERES OF THE LORENTZ SPACES $L_{w, 1}$
}

\author{
by N. L. CAROTHERS, S. J. DILWORTH $\dagger$ and D. A. TRAUTMAN
}

(Received 24 July, 1990)

We identify the extreme points of the unit sphere of the Lorentz space $L_{w, 1}$. This yields a characterization of the surjective isometries of $L_{w, 1}(0,1)$. Our main result is that every element in the unit sphere of $L_{w, 1}$ is the barycenter of a unique Borel probability measure supported on the extreme points of the unit sphere of $L_{w, 1}$.

1. Notation and terminology. For a measurable function $f$ defined on $(0, \infty)$ we define the distribution of $f$ by $d_{f}(t)=|\{x:|f(x)|>t\}|, 0<t<\infty(|A|$ denotes the Lebesgue measure of the set $A$ ), and the decreasing rearrangement of $f$ by $f^{*}(t)=\inf \left\{s>0: d_{f}(s) \leq\right.$ $t\}$. Following [5] we define the Lorentz space $L_{w, 1}(0, \infty)$ as the space of all (equivalence classes of) measurable functions $f$ on $(0, \infty)$ for which $\|f\|=\int_{0}^{\infty} f^{*}(t) w(t) d t<\infty$, where $w:(0, \infty) \rightarrow(0, \infty)$ is a strictly decreasing function satisfying $\lim _{t \rightarrow 0} w(t)=\infty, \lim _{t \rightarrow \infty} w(t)=0$, $\int_{0}^{1} w(t) d t=1$, and $\int_{0}^{\infty} w(t) d t=\infty . \quad L_{w, 1}$ is sometimes referred to as $\Lambda_{\phi}$ where $\phi(t)=\int_{0}^{t} w(s) d s, t \geq 0$. The fact that $w$ is strictly decreasing implies that $\phi$ is strictly concave.

For $M>0, L_{w, 1}(0, M)$ is the subspace of $L_{w, 1}(0, \infty)$ consisting of those functions which are supported on $[0, M]$. We shall write $L_{w .1}$ when the domain does not affect the argument.

$I(A)$ denotes the characteristic function of a set $A \subset[0, \infty)$. If $0<|A|<\infty$, we write $e(A)=I(A) / \phi(|A|)$ (so that $e(A)$ is of norm one in $\left.L_{w, 1}\right)$. $A^{c}$ denotes the complement of $A$, and $\{f>t\}$ denotes the set $\{s: f(s)>t\}$.

Given a Banach space $X, \mathrm{Ba}(X)$ denotes its closed unit ball. For a subset $B$ of $X$, conv $(B)$ denotes the convex hull of $B$.

Finally, we would like to thank Chris Lennard for some helpful discussions.

2. Preliminary results. Before we can prove our main result (Theorem 3.5), we need a description of the extreme points of $\mathrm{Ba}\left(L_{w, 1}\right)$. For the sequence space $l_{p, 1}[3]$ and the function space $L_{p, 1}$ [2], a characterization of the extreme points is well-known, but we do not know a reference for the general result. Because this general result (Lemma 2.1) will be needed later, we supply a proof below. As a consequence, we get a characterization of the surjective isometries of $L_{w, 1}$ (Theorem 2.3),

LEMMA 2.1 (cf. [2, Lemma 2.1]). If $f, g \in L_{w, 1}(0, \infty)$ satisfy $\|f+g\|=\|f\|+\|g\|$, then $(f+g)^{*}=f^{*}+g^{*}$.

Proof. Let $h(t)=f^{*}(t)+g^{*}(t)-(f+g)^{*}(t)$, and let $H(t)=\int_{0}^{t} h(s) d s$. Then $\int_{0}^{\infty} h(t) w(t) d t=\|f\|+\|g\|-\|f+g\|=0$, and $H(t) \geq 0$ by definition of the decreasing rearrangement. Integration by parts yields

$$
\int_{0}^{\infty} H(t) d(-w(t))=-\left.H(t) w(t)\right|_{0} ^{\infty}+\int_{0}^{\infty} h(t) w(t) d t=0+0=0 .
$$

† Supported in part by NSF DMS 8801731.

Glasgow Math. J. 34 (1992) 21-25. 
Since $H$ is continuous and $w$ is strictly decreasing, it follows that $H(t) \equiv 0$. This implies that $h(t)=0$ a.e.

We prove our result on extreme points for the space $L_{w, 1}(0, \infty)$, but the same proof works for $L_{w, 1}(0, M)$.

Proposition 2.2. Let $f \in L_{w, 1}$. Then $f$ is an extreme point of $\mathrm{Ba}\left(L_{w, 1}\right)$ if and only if $|f|=e(A)$ for some $A \subset(0, \infty)$ of finite, positive measure.

Proof. Suppose first that $f \in L_{w, 1}(0, \infty)$ and that there exists $A \subset(0, \infty)$ of finite, positive measure such that $|f|=e(A)$. Then $f^{*}=I(0,|A|) / \phi(|A|)$. If $f=g+h$ with $\|g\|+\|h\|=1$, then $f^{*}=g^{*}+h^{*}$ by Lemma 2.1. Since $g^{*}$ and $h^{*}$ are non-increasing, this implies that $g^{*}$ and $h^{*}$ are multiples of $f^{*}$. But then $|g|$ and $|h|$ must be multiples of $|f|$, and so $g$ and $h$ are multiples of $f$. Thus, $f$ is an extreme point of $\mathrm{Ba}\left(L_{w, 1}\right)$.

Now suppose that $|f|$ is not a multiple of $I(A)$ for any $A \subset(0, \infty)$ of finite, positive measure. Then there exists $\lambda>0$ such that if $A=\{|f|>\lambda\}$, we have $|A|>0$ and $\left\|f I\left(A^{c}\right)\right\|>0$. Let $g=(f-\lambda \operatorname{sgn}(f)) I(A)$, and let $h=f-g$. Clearly, $\|g\|>0,\|h\|>0$, and $f \neq g /\|g\|$. But $f^{*}=g^{*}+h^{*}$, and so $\|f\|=\|g\|+\|h\|$. Since $f=\|g\|(g /\|g\|)+$ $\|h\|(h /\|h\|)$, it follows that $f$ is not an extreme point of $\mathrm{Ba}\left(L_{w, 1}\right)$.

We now characterize the surjective isometries of $L_{w, 1}(0,1)$. Our proof is based on the description of the isometries of $L_{p}(0,1), 1<p<\infty$ [6, pp. 415-418]. We present only a sketch of the proof, but the details are easy to check.

THEOREM 2.3. Let $T$ be a surjective isometry of the space $L_{w, 1}(0,1)$. Then there exists $a \pm 1$-valued Borel measurable function $\varepsilon$ and a Borel measurable map $\sigma$ from $[0,1]$ to $[0,1]$ which is measure-preserving (i.e., $\left.\left|\sigma^{-1}(A)\right|=|A|\right)$ such that

$$
(T f)(t)=\varepsilon(t) f(\sigma(t)), \quad 0 \leq t \leq 1 .
$$

Proof. Since $T$ is a surjective isometry, $T$ maps the set of extreme points of $\mathrm{Ba}\left(L_{w, 1}\right)$ onto itself. By Proposition 2.2, we know that for every Borel set $A \subset[0,1]$ there is a Borel set $A^{\prime}$ such that $|T(e(A))|=e\left(A^{\prime}\right)$. Define a mapping $\psi$ from $\mathscr{B}$, the collection of Borel sets of $[0,1]$, into $\mathscr{B} / \mathcal{N}$, where $\mathcal{N}$ is the collection of Borel sets of measure zero, by setting $\psi(A)=A^{\prime}$. It is easy to check that $\psi$ sends disjoint sets to disjoint sets, that $\psi\left(\bigcup_{n=1}^{\infty} A_{n}\right)=\bigcup_{n=1}^{\infty} \psi\left(A_{n}\right)$ for disjoint sets $\left(A_{n}\right)$, and that $|\psi(A)|=|A|$. In particular, $\psi([0,1])=[0,1]$. Arguing now as in [6, p. 417], there exists a Borel mapping $\sigma$ on $[0,1]$ such that $\psi(A)=\sigma^{-1}(A)$ for every $A \in \mathscr{B}$. Finally, define a \pm 1 -valued Borel measurable function $\varepsilon$ by $\varepsilon=T(I(0,1))$. The conclusion of the theorem now follows easily.

3. A uniqueness theorem. It is well-known that $L_{w, 1}$ is a separable dual space [5]. One consequence of this fact is that for every $f \in L_{w, 1}$ with $\|f\|=1$, there is a probability measure $\mu$ on $\mathrm{Ba}\left(L_{w, 1}\right)$ which is supported on the extreme points of $\mathrm{Ba}\left(L_{w, 1}\right)$ such that $f$ is the barycenter of $\mu$ :

$$
f=\int_{\mathrm{Ba}\left(L_{w, 1}\right)} x d \mu(x)
$$


Our goal in this section is to show that this representation of $f$ is unique. We begin with two technical lemmas. The proof of the first is straightforward (see e.g. [1, Section 2.7]).

LEMMA 3.1. Let $f$ be a nonnegative, locally integrable function on $(0, \infty)$. For each $t>0$ there exists $A \subset(0, \infty)$ such that $|A|=t$ and $\int_{0}^{t} f^{*}=\int_{A} f$. Moreover, any such $A$ is necessarily of the form $\{f>\lambda\} \cup C$, where $C \subset\{f=\lambda\}$, for some $\lambda \geq 0$.

LEMMA 3.2. Let $f$ and $g$ be nonnegative functions in $L_{w, 1}$ such that $\|f+g\|=$ $\|f\|+\|g\|$, and let $B=\{f>\lambda\}$, where $\lambda>0$. Then $\underset{B}{\operatorname{ess}} \inf g \geq \underset{B^{c}}{\operatorname{ess} \sup } g$.

Proof. Let $t=|B|$. Applying Lemma 3.1 to $f+g$, there exists $A \subset(0, \infty)$ such that $|A|=t$ and $\int_{0}^{t}(f+g)^{*}=\int_{A}(f+g)$. By Lemma 2.1, $(f+g)^{*}=f^{*}+g^{*}$, so $\int_{0}^{t}\left(f^{*}+g^{*}\right)$ $=\int_{A}(f+g)$. This implies that $\int_{0}^{t} f^{*}=\int_{A} f$ and $\int_{0}^{t} g^{*}=\int_{A} g$. By Lemma 3.1 we have $A$ $=B=\{f>\lambda\}$, and there exists an $\alpha>0$ such that $A=\{g>\alpha\} \cup C$, where $C \subset\{g=\alpha\}$. The conclusion of the lemma follows immediately.

Let $G$ denote the collection of extreme points of $\mathrm{Ba}\left(L_{w, 1}\right)$ and let $\mu$ be a regular Borel probability measure on $\mathrm{Ba}\left(L_{w, 1}\right)$ such that $\mu(G)=1$. Let us say that an extreme point $e$ belongs to the support of $\mu$ if $\mu(U)>0$ for every norm-open neighborhood $U$ of $e$, and let $H$ denote the collection of extreme points in the support of $\mu$. Then $H$ is a $G_{\delta}$-set in $L_{w, 1}$ and $G \backslash H$ is contained in a union of $\mu$-null open sets. Since $L_{w, 1}$ is separable it is a Lindelöf space, and so $\mu(G \backslash H)=0$, whence $\mu(H)=1$. We are now in a position to give the main technical ingredient in the proof of our theorem.

LemMa 3.3. Suppose that $f$ is a nonnegative function on $(0, \infty)$, that $\|f\|=1$, and that $f$ is the barycenter of a Borel probability measure $\mu$ as described above. Then every extreme point $e$ in the support of $\mu$ is of the form $e(E)$, where $E=\{f \geq \lambda\}$ or $E=\{f>\lambda\}$, for some $\lambda \geq 0$.

Proof. It is clear that every extreme point in the support of $\mu$ is nonnegative, and so $e$ is of the form $e(E)$ for some $E \subset(0, \infty)$. If $E$ is not of the form described in the statement of the lemma, then there exist a $\lambda>0$ and disjoint sets $A$ and $B$ of positive Lebesgue measure such that $A \subset\{f \geq \lambda\} \cap E^{c}$ and $B \subset\{f \leq \lambda\} \cap E$. Thus $\left.e\right|_{A}=0$ and $\left.e\right|_{B}=\rho$ for some $\rho>0$. Let $\varepsilon>0$; since $e$ lies in the support of $\mu$ there exists a neighborhood $U$ of $e$ of diameter less than $\varepsilon$ such that $\mu(U)>0$. Let $g$ and $h$ be defined by $g=\int_{U} x d \mu(x)$ and $h=\int_{U^{c}} x d \mu(x)$. Now $g / \mu(U)$ and $e$ are close in measure, since $\|g / \mu(U)-e\|<\varepsilon$. By choosing $\varepsilon>0$ sufficiently small we may assume that there exist $A^{\prime} \subset A$ and $B^{\prime} \subset B$ of positive Lebesgue measure such that $g / \mu(U)>\rho / 4$ on $A^{\prime}$ and $g / \mu(U)>3 \rho / 4$ on $B^{\prime}$. Recall that $A^{\prime} \subset A \subset\{f \geq \lambda\}$ and $B^{\prime} \subset B \subset\{f \leq \lambda\}$, and that $f=g+h$. Thus, for almost all $a^{\prime} \in A^{\prime}$ we have

$$
h\left(a^{\prime}\right)=f\left(a^{\prime}\right)-g\left(a^{\prime}\right) \geq \lambda-\rho \mu(U) / 4,
$$

and for almost all $b^{\prime} \in B^{\prime}$ we have

and so

$$
h\left(b^{\prime}\right)=f\left(b^{\prime}\right)-g\left(b^{\prime}\right) \leq \lambda-3 \rho \mu(U) / 4,
$$

$$
\underset{B^{\prime}}{\operatorname{ess} \inf } h \leq \underset{A^{\prime}}{\operatorname{ess} \sup } h-\rho \mu(U) / 2 \text {. }
$$


To derive a contradiction, first observe that $B^{\prime} \subset\{g>3 \rho \mu(U) / 4\}$ and $A^{\prime} \subset\{g<$ $\rho \mu(U) / 4\}$. Also, $\|g+h\|=\|g\|+\|h\|$ since $f$ and the support of $\mu$ both lie in the unit sphere of $L_{w, 1}$. Combined with Lemma 3.2 it now follows that $\underset{B^{\prime}}{\operatorname{ess}} \inf h \geq \underset{A^{\prime}}{\operatorname{ess} \sup } h$, which contradicts $(*)$.

Lemma 3.4. Let $f \in L_{w, 1}$ with $\|f\|=1$. Then $f^{*}$ is the barycenter of a unique Borel probability measure supported on the extreme points of $\mathrm{Ba}\left(L_{w, 1}\right)$.

Proof. By Lemma 3.3, the support of every $\mu$ for which $f^{*}$ is a barycenter is contained in $S=\{e(0, u): u>0\}$. The homeomorphism $u \mapsto e(0, u)$ from $(0, \infty)$ onto $S$ induces a bijection between the regular Borel probability measures supported on $S$ and those on $(0, \infty)$. Suppose $\mu$ corresponds to $\hat{\mu}$ under this bijection. Then

$$
f^{*}(u)=\int_{(u, \infty)} \frac{d \hat{\mu}}{\phi}
$$

for all $u$ by the right continuity of $f^{*}$. Now every regular Borel measure on $(0, \infty)$ is the Lebesgue-Stieltjes measure defined by its indefinite integral $[4$, p. 331]; thus $(1 / \phi) d \hat{\mu}=$ $d\left(-f^{*}\right)$, and $d \hat{\mu}=\phi d\left(-f^{*}\right)$. The uniqueness of $\mu$ follows at once.

THEOREM 3.5. Let $f \in L_{w, 1}$ with $\|f\|=1$. Then $f$ is the barycenter of a unique Borel probability measure supported on the extreme points of $B a\left(L_{w, 1}\right)$.

Proof. The mapping $g \mapsto g . \varepsilon$, where $\varepsilon$ is a \pm 1 -valued measurable function, defines an isometry from $L_{w, 1}$ onto $L_{w, 1}$, and hence we may assume that $f$ is non-negative. Given a nonnegative function $g \in L_{w, 1}$, define the set $S(g)$ by

$$
S(g)=\{e(\{g>\lambda\}): \lambda>0\} \cup\{e(\{g \geq \lambda\}): \lambda>0\} .
$$

Define a map $T$ from $S(f)$ onto $S\left(f^{*}\right)$ by $T(e(\{f>\lambda\}))=e\left(\left\{f^{*}>\lambda\right\}\right), T(e(\{f \geq \lambda\}))$ $=e\left(\left\{f^{*} \geq \lambda\right\}\right)$. By linearity, $T$ extends from $\operatorname{conv}(S(f))$ onto $\operatorname{conv}\left(S\left(f^{*}\right)\right)$. Since $f$ and $f^{*}$ have the same distribution, it is easily verified that $T$ is an affine isometry. So, $T$ extends to an affine isometry from the closed convex hull of $S(f)$ onto the closed convex hull of $S\left(f^{*}\right)$ with $T(f)=T\left(f^{*}\right)$. By Lemma 3.3, if $f$ is the barycenter of a Borel probability measure $\mu$ of the required type, then the support of $\mu$ is contained in $S(f)$. Under the affine isometry $T$, the measure $\mu$ corresponds to a measure $\mu^{*}$ whose barycenter is $f^{*}$. By Lemma $3.4, \mu^{*}$ is unique, whence $\mu$ is unique.

The proof of Theorem 3.5 implies the following corollary.

Corollary 3.6. Suppose $f \in L_{w, 1}$ with $\|f\|=1$. Then $f$ admits a representation of the form $f=\sum_{n=1}^{\infty} \lambda_{n} e_{n}$, where $\lambda_{n} \geq 0, \sum_{n=1}^{\infty} \lambda_{n}=1$, and each $e_{n}$ is an extreme point of $\mathrm{Ba}\left(L_{w, 1}\right)$, if and only if $d\left(f^{*}\right)$ is purely atomic (that is, if and only if $f^{*}$ is a saltus function; $c f ., e . g$, [4, p. 335]).

REMARK. Of course, if $\|f\|<1$, then a representing measure supported on the extreme points will no longer be unique. In fact, it is easy to see that $f$ can always be expressed in the form $f=\sum_{n=1}^{\infty} \lambda_{n} e_{n}$ as described in Corollary 3.6. 


\section{REFERENCES}

1. C. Bennett and R. C. Sharpley, Interpolation of Operators (Academic Press, 1988).

2. N. L. Carothers and B. Turett, Isometries on $L_{p, 1}$, Trans. Amer. Math. Soc. 297 (1986), 95-103.

3. W. J. Davis, Positive bases in Banach spaces, Rev. Roumaine Math. Pures Appl. 16 (1971), 487-492.

4. E. Hewitt and K. Stromberg, Real and Abstract Analysis. (Springer-Verlag, 1975).

5. J. Lindenstrauss and L. Tzafriri, Classical Banach Spaces II: Function Spaces, Ergebnisse Math. 2. Folge 97 (Springer-Verlag, 1979).

6. H. L. Royden, Real Analysis, 3rd. Ed. (Macmillan, 1988).

N. L. Carothers:

Bowling Green State University

Bowling Green

Оніо 43403

U.S.A.
S. J. Dilworth:

University of South Carolina

Columbia

South Carolina 29208

U.S.A.

D. A. Trautman:

The Citadel

Charleston

South Carolina 29409

U.S.A. 